Research Article

\title{
Cognitive Training Attenuates Decline in Physical Function Across 10 Years
}

\author{
Briana N. Sprague, PhD, ${ }^{1, *, 0}$ Christine B. Phillips, PhD, ${ }^{2}$ and Lesley A. Ross, PhD ${ }^{1,3}$ \\ 'Department of Human Development and Family Studies, The Pennsylvania State University, University Park. ${ }^{2}$ Atria Senior \\ Living, Louisville, Kentucky. ${ }^{3}$ Department of Psychology, Clemson University, South Carolina.
}

Briana N. Sprague is now a postdoctoral fellow at the Department of Epidemiology, University of Pittsburgh.

Lesley A. Ross is now at the Department of Psychology, Clemson University.

*Address correspondence to: Briana N. Sprague, PhD, The Pennsylvania State University, 119 Health and Human Development Building, University Park, PA 16802. E-mail: sprague.briana@gmail.com

Received: September 19, 2019; Editorial Decision Date: May 18, 2020

Decision Editor: Vanessa Taler, PhD

\begin{abstract}
Objectives: Poor physical function is associated with negative health and cognitive outcomes. Although nine studies demonstrated that cognitive training reduces age-related declines in physical function, only one study has examined the effects beyond immediate posttest changes. The first aim of this study was to assess the impact of three cognitive training programs on physical function measures across 10 years and the second aim was to examine whether baseline cognitive self-efficacy or depressive symptoms moderated training effects.

Method: Using data from the Advanced Cognitive Training for Independent and Vital Elderly (ACTIVE) randomized controlled trial, older adults in a no-contact control condition $(n=698)$ were compared to those receiving processing speed $(n=702)$, memory $(n=703)$, or reasoning $(n=694)$ training. Intention-to-treat (ITT) and dosage analyses were conducted for grip strength and Turn 360. Participants were followed up to 10 years posttest.

Results: There were no significant ITT effects of processing speed, memory, or reasoning training assignment to any physical function outcome $(p>.05)$. Dosage models indicated that there were small age-related attenuation effects in Turn 360 decline with more processing speed training $(b=-.011, p<.001)$, memory training $(b=-.011, p<.001)$, and reasoning training $(b=-.012, p<.001)$. There was no significant transfer to grip strength. These training gains were greater for those with more baseline depressive symptoms who received more processing speed training $(b=-.001, p<.001)$.

Discussion: This is the first study to demonstrate the effects of cognitive training to complex physical function across 10 years.
\end{abstract}

Keywords: Cognitive interventions, Grip strength, Healthy older adults, Lower limb function

Physical function is critical for maintaining independence in older adulthood (Cesari et al., 2004), and its degradations are associated with myriad poor health measures, including loss of functional independence (Cesari et al., 2004), increased risk of hospitalization (Cesari et al., 2004), disability (Buchman et al., 2016), poorer cognitive function (Spedden, Malling, Andersen, \& Jensen, 2017), and mortality (Nofuji et al., 2016). Emphasizing impairment prevention is important because it may reduce costly downstream outcomes such as falls (Mangani et al., 2008), everyday function disability (Gobbens \& van Assen, 2014), and hospital length of 
stay (van Aalst, Oosterhof, Nijhuis-van der Sanden, \& Schreurs, 2014).

The most common behavioral intervention for physical function is balance-related exercises (Chou, Hwang, \& Wu, 2012). However, some argue that exercise may be necessary but insufficient to prevent physical function decline or disability (Keysor \& Brembs, 2011). Furthermore, older adults with poor physical function may be disinclined to participate due to barriers such as perceived or previous falling or injury (Murphy, Dubin, \& Gill, 2003). This is problematic as fear of falls (Choi, Jeon, \& Cho, 2017), previous falls (Kronzer et al., 2016), and decreased exercise (Paterson \& Warburton, 2010) are risk factors for subsequent functional decline, leading to a cycle of excess disability. Additionally, without continual exercise engagement to maintain training gains (Mujika \& Padilla, 2000), longterm physical function benefits may be limited. Because older adults tend to cease engagement within 1 year of exercise intervention (Henderson et al., 2018), it is important to identify non-exercise interventions that do not require continual engagement but have long-term benefits.

Although exercise is the most promising avenue for physical function maintenance (Marusic \& Grosprêtre, 2018), some cognitive training programs have positively impacted physical function in older adults across 12 studies (SmithRay et al., 2015) (Azadian, Majlesi, \& Jafarnezhadgero, 2018; Azadian, Torbati, Kakhki, \& Farahpour, 2016; Li et al., 2010; Marusic et al., 2015; Marusic, Verghese, \& Mahoney, 2018; Ng et al., 2015; Ross, Sprague, Phillips, O'Connor, \& Dodson, 2018; Smith-Ray et al., 2015; SmithRay, Irmiter, \& Boulter, 2016; Smith-Ray, MakowskiWoidan, \& Hughes, 2014; Verghese, Mahoney, Ambrose, Wang, \& Holtzer, 2010). Generally, processing speed training (also Useful Field of View, UFOV, speed of processing or divided attention training) transfers to maintained complex lower limb functions, but recent work extends this to memory and reasoning training and gross and fine motor upper limb function across 5 years (Ross, Sprague, et al., 2018). One way cognitive training is posited to impact physical function is compensation, or additional recruitment of neural resources to respond to tasks with high cognitive demand (Cabeza et al., 2018). For example, brain regions associated primarily with processing speed and reasoning, for example, dorsolateral prefrontal cortex, are activated when older adults walk (Holtzer, Epstein, Mahoney, Izzetoglu, \& Blumen, 2014; Pelicioni, Tijsma, Lord, \& Menant, 2019), indicating that cognitive and physical function are related, even when processing speed or other cognitive tasks are not explicitly performed. These associations are more pronounced in older adults (Spedden et al., 2017). It is assumed that as a result, cognitive training, especially processing speed and reasoning training, may confer benefits to physical function. It is notable that memory training does have promise for attenuating declines in physical function, but only one study has implemented strategy-based memory training (Ross, Sprague, et al., 2018).
Despite emerging, consistent evidence of transfer from cognitive training to physical function (Azadian et al., 2016, 2018; Li et al., 2010; Marusic et al., 2015, 2018; Ng et al., 2015; Ross, Sprague, et al., 2018; Smith-Ray et al., 2015, 2014, 2016; Verghese et al., 2010), there are remaining gaps in the extant literature. A major limitation is that all but one trial (Ross, Sprague, et al., 2018) had small samples of participants (e.g., <100). Some of these studies also drew from at-risk populations rather than low-risk, community-dwelling participants (Smith-Ray et al., 2015). While informative about the effectiveness of training for at-risk populations, the tradeoff is unknown generalizability to a wider community-dwelling sample. Additionally, only one study to date assessed long-term physical function, suggesting declines may be attenuated up to 5 years post-training (Ross, Sprague, et al., 2018). Processing speed training maintained everyday function measures such as reductions in dementia risk (Edwards et al., 2017), maintained reported instrumental activities of daily living (along with reasoning training) (Rebok et al., 2014), and maintained driving mobility (Ross, Freed, Edwards, Phillips, \& Ball, 2017) across a decade. Therefore, training effects may extend to physical function across at least 10 years.

\section{Psychosocial Moderators of Training Gains}

Psychosocial factors such as greater self-efficacy and fewer depressive symptoms have well-documented relationships with better physical (Briggs, Carey, Kenney, \& Kennelly, 2018) and cognitive functions (Zahodne, Watson, Seehra, \& Martinez, 2018). Evidence suggests those with greater cognitive self-efficacy are not more responsive to processing speed training (Sharpe, Holup, Hansen, \& Edwards, 2014); however, higher cognitive self-efficacy is associated with greater responsiveness to reasoning training (Payne et al., 2012). This suggests that one's beliefs in their abilities may influence other factors such as motivation to persevere with a task (or training program) and by extension impact the responsiveness to treatment (Beaudoin \& Desrichard, 2017). Additionally, limited evidence suggests that those with greater depressive symptoms have poorer baseline performance but similar responsiveness to memory training (Lohman et al., 2013). The impact of baseline depressive symptoms on processing speed or reasoning training, as well as the impact of both baseline psychosocial factors on physical function training gains, remains unknown.

\section{Aims and Hypotheses}

The first aim of this study was to examine the 10-year effects of three cognitive training programs (processing speed, memory, or reasoning) compared to a no-contact control group on physical function (grip strength and Turn 360) in a large, community-dwelling sample of older adults. Similar to prior work across 5 years (Ross, Sprague, et al., 2018), we hypothesized that the age-related declines in Turn 360 would be attenuated in all training groups, and 
that reasoning training would continue to be associated with attenuated declines in both measures (Ross, Sprague, et al., 2018). The second aim was to identify which baseline moderators impacted training effects. We had no strong directional a priori hypotheses due to the sparse literature but anticipated there would be moderated effects of training on both physical function measures by psychosocial factors.

\section{Method}

\section{Participants}

This study used secondary data from the Advanced Cognitive Training for Independent and Vital Elderly (ACTIVE) study, a multi-site, randomized clinical trial investigating the effect of three cognitive training programs on health and functional outcomes across a 10 -year period. Physical function was an a priori identified secondary outcome of the ACTIVE trial but current analyses were not preregistered. Inclusion criteria were: age 65 or older, visual acuity $\geq 20 / 50$, Mini-Mental State Examination score $\geq 23$, no health conditions associated with cognitive impairment, verbal communication skills, no difficulties performing basic activities of daily living, and no recent participation in cognitive training. Eligible participants $(N=2,802)$ completed a baseline assessment and then were randomly assigned to a cognitive training (to processing speed, memory, or reasoning training) or no-contact control condition. At baseline, participants were an average of 73.60 years old $(S D=5.90)$, were predominantly women $(n=2,121 ; 75.83 \%)$, reported an average of 13.5 years of education $(S D=2.70)$, and were predominantly White $(n=2,051,73.33 \%)$. Sample descriptive statistics for each arm are presented in Table 1. Extensive details about ACTIVE can be found elsewhere (Jobe et al., 2001), ClinicalTrials.gov Identifier NCT00298558.

\section{Study Design and Procedures}

Baseline assessments for the ACTIVE study were conducted between 1998 and 1999. Prior to randomization, baseline cognitive and physical function, lifestyle factors, and health assessments were conducted. The initial cognitive training interventions consisted of ten 60- to 75-min sessions administered over 6 weeks. Follow-up assessments were conducted approximately 2 months (posttest), 1 year, 2 years, 3 years, 5 years, and 10 years after baseline testing. Grip strength and Turn 360 were not assessed at the immediate posttest or 1-year post-intervention. Compliant training arm participants (i.e., completed at least eight of 10 sessions) were further randomized to either receive four booster sessions prior to assessments in years 1 and 3 (totaling eight possible booster sessions) or receive no booster training.

\section{Processing speed training arm}

This was process-, computer-based training that focused on improving the speed at which participants could correctly

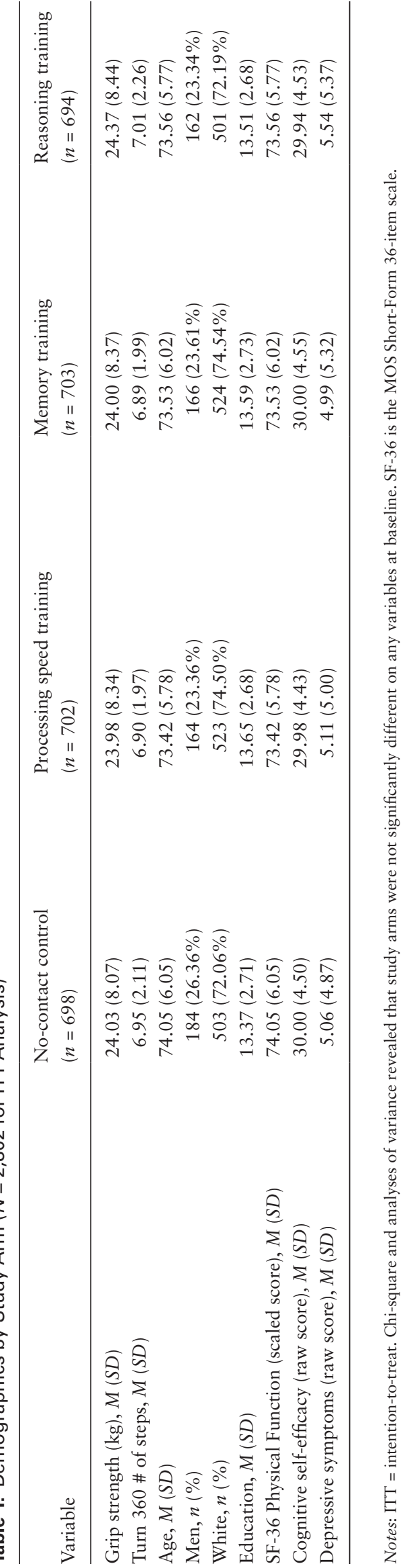


identify increasingly complex visual information. For each task, the score was the shortest presentation time needed to perform the task correctly $75 \%$ of the time.

\section{Memory training arm}

This was a strategy-based pencil-and-paper training designed to improve verbal episodic memory using mnemonic strategies. Training included practice and feedback organizing materials into meaningful categories, such as lists of errands or grocery shopping (Jobe et al., 2001).

\section{Reasoning training arm}

This was a strategy-based pencil-and-paper training that focused on improving problem solving and included practice and feedback on identifying patterns or sequences. For example, training activities included finding the pattern in a series of repeating letters or identifying dosage patterns of mediations (Jobe et al., 2001).

\section{No-contact control arm}

Participants randomized to the no-contact control arm came to the study site for all assessments, and no intervention was conducted.

\section{Measures}

\section{Physical function}

Grip strength, a measure of gross upper limb muscle strength, was assessed using the Jamar hydraulic hand dynamometer (Lafayette Instruments). Participants were instructed to squeeze as hard as they comfortably could in their dominant hand, and the assessment was completed twice. Participants reporting recent worsening of pain or arthritis in their wrists, had tendonitis, or hand or arm surgery during the prior three months did not complete the grip strength protocol. Scores indicated the output strength in kilograms rounded to the nearest whole number and were averaged across the two trials to yield a composite measure of grip strength. Scores were baseline-adjusted by subtracting the baseline mean and dividing by the baseline standard deviation.

Turn 360, a measure of complex lower limb function, was assessed (Gill, Williams, \& Tinetti, 1995; SteinhagenThiessen \& Borchelt, 1999; Tager, Swanson, \& Satariano, 1998). Participants were instructed to turn in a complete circle as quickly and safely as possible. If necessary, walking aids were permitted. Scores were the average number of steps across two trials. Scores were baseline-adjusted by subtracting the baseline mean and dividing by the baseline standard deviation. Higher scores indicated worse performance. See Supplementary Table 1 for the unadjusted means of grip strength and Turn 360 across the study period stratified by intervention group.

\section{Training}

Intention-to-treat (ITT) multilevel models assessed the effect of randomization on training regardless of adherence. Training groups were separately compared against the no-contact control group. In addition, dosage-received models where only those who were compliant in the initial trial (i.e., at least 8 of 10 sessions) or the no-contact control group were analyzed to examine whether receiving the treatment impacted physical function. Dosage was assessed as number of training sessions completed as a time-varying measure. Follow-up analyses with only those receiving at least one booster (i.e., at least 11 training sessions) were completed to assess whether training gains were similar to the main dosage analyses. Participants were randomized to receive booster training if they were compliant in the initial trial; $60 \%$ of the compliant sample in each training arm were randomized to receive the booster, while the remaining $40 \%$ did not.

\section{Baseline psychosocial moderators}

All baseline moderators evaluated whether baseline status attenuated 10-year declines in physical function slopes. Cognitive self-efficacy was assessed using the Personality in Intellectual Contexts-36 item (PIC-36) internal locus of control subscale (Lachman, Baltes, Nesselroade, \& Willis, 1982), which consisted of six items assessing one's ability to (re)learn unfamiliar materials. All items were scored on a 1 (strongly agree) to 6 (strongly disagree) Likert scale. Items were summed for a summary score then mean centered for the analyses. The summary scores ranged from 8 to 36; higher scores indicated higher cognitive self-efficacy.

Depressive symptoms were assessed using the Center for Epidemiological Studies-Depression 12-item questionnaire (CES-D-12) (Radloff, 1977). Participants were asked to describe how they felt or behaved during the prior week. All items were scored on a 0 (never) to 3 (5-7 days) Likert scale. Items were summed for a summary score then mean centered for the analyses. The summary scores ranged from 0 to 34 (theoretical maximum $=36$ ); higher scores indicated more depressive symptoms.

\section{Covariates}

Self-reported baseline age, gender $($ woman $=0 ;$ man $=1)$, race (non-White $=0$ or White $=1$ ), and years of formal $e d u$ cation (0-20) were included as covariates because older age, women, non-White race, and lower educational attainment have been associated with lower physical function. Baseline age and education were grand mean centered at baseline such that higher scores indicated older age and more education, respectively. Additionally, baseline self-reported physical function using the SF-36 physical function subscale was included. Scores were standardized based on the SF-36 scoring manual and then centered based on the sample mean; higher scores indicated better self-reported function.

\section{Analytic Strategy}

Chi-square and analyses of variance were used to test baseline differences; there were no differences in any characteristics of interest by intervention group. All multilevel models were 
estimated using restricted maximum likelihood estimation with an unstructured covariance matrix and were conducted with SPSS, version 25 (IBM Corporation). All estimates were unstandardized, and significance was evaluated at $p<.05$. Outcome variables were standardized by first subtracting the baseline mean and dividing by the baseline standard deviation. Time was scaled to reflect the number of months since the baseline assessment. Similar to prior work (Ross, Sprague, et al., 2018), base models were developed using the control arm of the study $(n=698)$ and included all demographics, subjective physical function, and Time* Covariate (including time ${ }^{2}$ ) interactions. Nonsignificant covariates and interactions were removed, resulting in different covariates for each physical function outcome measure. The same covariates were also used for dosage analyses. Participants completing at least eight of the initial sessions were included in the dosage analyses, and number of sessions completed was time-varying dosage. Unadjusted effect sizes (Cohen's $d$ ) are presented. Lastly, all baseline psychosocial moderation and bodily pain analyses were analyzed separately in order to simplify interpretation of the results.

\section{Results}

\section{Base Models}

The base model using the control group for grip strength indicated that younger age (estimate $=-0.04, p<.001$ ), men (estimate $=-1.49, p<.001$ ), non-White participants (estimate $=0.36, p<.001$ ), and those with better baseline self-reported physical function (estimate $=0.01, p<.001$ ) had better grip strength. Grip strength significantly weakened over time (estimate $=-0.004, p<.001$ ). A significant time $^{2}$ interaction suggested grip strength declines accelerated over time (estimate $<0.001, p<.001$ ). There were 200 processing speed training, 196 memory training, 208 reasoning training, and 183 no-contact control participants with grip strength data at year 10 .

The base model for Turn 360 using the control group indicated that younger age (estimate $=0.041, p<.001$ ), White participants (estimate $=-0.16, p=.01$ ), and those with better physical function (estimate $=-0.01, p<.001$ ) had better Turn 360. Over time, Turn 360 scores significantly declined (estimate $=0.007, p<.001$ ). Lastly, there was a significant Age*Time interaction such that over time, those who were older had accelerated decline (estimate $<0.001, p=.002$ ). There were 229 processing speed training, 215 memory training, 227 reasoning training, and 201 no-contact control participants with Turn 360 data at year 10 .

\section{Grip Strength}

\section{Aim 1: ITT models}

Assignment to processing speed (estimate $<0.001$, $p=.454)$, memory (estimate $<0.001, p=.647$ ), or reasoning training (estimate $=0.001, p=.251$ ) were not significantly associated with better grip strength relative to the control group. Additionally, assignment to processing speed (estimate $=<0.001, p=.478)$, memory (estimate $=<-0.001$, $p=.684$ ), or reasoning training (estimate $=<0.001$, $p=.984$ ) were not associated with differences in accelerated grip strength weakness over time.

\section{Aim 1: dosage models}

More processing speed (estimate $=<-0.001, S E=0.002$; $d=0.104$ ), memory (estimate $=<-0.001, S E=0.002 ; d=0.053$ ), and reasoning (estimate $=<0.001, S E=0.002 ; d=0.097$ ) training were not significantly associated with grip strength relative to the control group ( $p>.05$; Table 2$)$. The same pattern of results held when constraining the analyses to those who received booster sessions only.

\section{Aim 2: psychosocial moderators}

Because there were no significant ITT or dosage models for grip strength across 10 years, moderation analyses were not completed for grip strength.

\section{Turn 360}

\section{Aim 1: ITT models}

Assignment to processing speed (estimate $=0.001, p=.133$ ), memory (estimate $<0.001, p=.867$ ), or reasoning training (estimate $=0.001, p=.186$ ) were not significantly associated with better Turn 360 relative to the control group.

\section{Aim 1: dosage models}

More processing speed (estimate $=-0.011, S E=0.003$; $d=0.216$ ), memory (estimate $=-0.011, S E=0.003$; $d=0.057$ ) , and reasoning (estimate $=-0.012, S E=0.003$; $d=0.210)$ training were associated with better Turn 360 relative to the control group $(p<.001$; Table 3$)$. The same pattern of results held when constraining analyses to only those who received booster training.

\section{Aim 2: psychosocial moderators}

Baseline cognitive self-efficacy did not moderate the relationship between dosage and Turn 360 for processing speed, memory, or reasoning training $(p>.05)$. Baseline depressive symptoms moderated the relationship between dosage and Turn 360 in the processing speed (estimate $=-0.001, p<$ $.05)$ group only, indicating that the benefit of additional training was greatest among those with more baseline depressive symptoms. See Supplementary Table 1 for the unadjusted means across time. There was no moderation in memory or reasoning training $(p>.05)$.

\section{Discussion}

Cognitive training is increasingly viewed as a viable intervention strategy for older adult physical function (Azadian et al., 2016, 2018; Li et al., 2010; Marusic \& Grosprêtre, 2018; Marusic et al., 2015, 2018; Ng et al., 2015; Ross, Sprague, et al., 2018; Smith-Ray et al., 2015, 2016; Verghese 


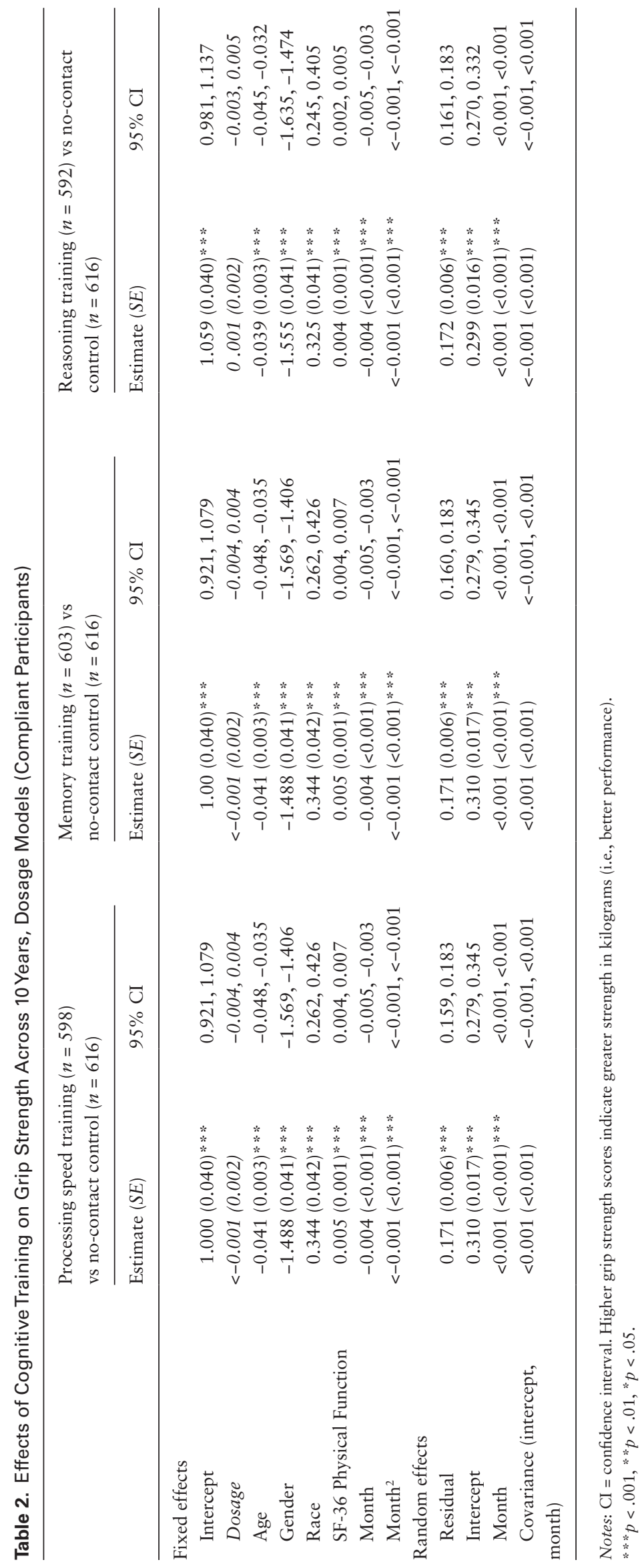


Table 3. Effects of Cognitive Training on Turn 360 Across 10 Years, Dosage Models (Compliant Participants)

\begin{tabular}{|c|c|c|c|c|c|c|}
\hline & \multicolumn{2}{|c|}{$\begin{array}{l}\text { Processing speed training }(n=684) \\
\text { vs no-contact control }(n=681)\end{array}$} & \multicolumn{2}{|c|}{$\begin{array}{c}\text { Memory training }(n=688) \\
\text { vs no-contact control }(n=681)\end{array}$} & \multicolumn{2}{|c|}{$\begin{array}{l}\text { Reasoning training }(n=678) \\
\text { vs no-contact control }(n=681)\end{array}$} \\
\hline & Estimate $(S E)$ & $95 \% \mathrm{CI}$ & Estimate $(S E)$ & $95 \% \mathrm{CI}$ & Estimate $(S E)$ & $95 \% \mathrm{CI}$ \\
\hline \multicolumn{7}{|l|}{ Fixed effects } \\
\hline Intercept & $0.305(0.030) * * *$ & $0.256,0.364$ & $0.305(0.030)^{* * *}$ & $0.246,0.364$ & $0.312(0.030) * * *$ & $0.253,0.371$ \\
\hline Dosage & $-0.011(0.003) * * *$ & $-0.016,-0.006$ & $-0.011(0.003) * * *$ & $-0.016,-0.006$ & $-0.012(0.003) * * *$ & $-0.017,-0.006$ \\
\hline Age & $0.056(0.004) * * *$ & $0.047,0.064$ & $0.055(0.004) * * *$ & $0.047,0.064$ & $0.055(0.004) * * *$ & $0.047,0.064$ \\
\hline Race & $-0.114(0.047)^{*}$ & $-0.207,-0.021$ & $-0.114(0.047)^{*}$ & $-0.207,-0.021$ & $-0.131(0.048)^{* *}$ & $-0.225,-0.037$ \\
\hline $\begin{array}{l}\text { SF-36 Physical } \\
\text { Function }\end{array}$ & $-0.012(0.001)^{* * * *}$ & $-0.013,-0.010$ & $-0.012(0.001) * * *$ & $-0.013,-0.010$ & $-0.012(0.001)^{* * * *}$ & $-0.013,-0.010$ \\
\hline Month & $0.007(<0.001)^{* * * *}$ & $0.006,0.008$ & $0.007(<0.001)^{* * * *}$ & $0.006,0.008$ & $0.007(<0.001) * * *$ & $0.006,0.008$ \\
\hline Age* Month & $<0.001(<0.001)^{* * *}$ & $<0.001,<0.001$ & $<0.001(<0.001)^{* * *}$ & $<0.001,<0.001$ & $<0.001(<0.001)^{* * * *}$ & $<0.001,<0.001$ \\
\hline \multicolumn{7}{|l|}{ Random effects } \\
\hline Residual & $0.410(0.013) * * *$ & $0.386,0.436$ & $0.410(0.013)^{* * * *}$ & $0.386,0.436$ & $0.417(0.013) * * *$ & $0.393,0.443$ \\
\hline Intercept & $0.433(0.028) * * *$ & $0.381,0.492$ & $0.433(0.028) * * *$ & $0.382,0.492$ & $0.445(0.029) * * *$ & $0.392,0.505$ \\
\hline Time & $<0.001(<0.001)^{* * *}$ & $<0.001,<0.001$ & $<0.001(<0.001)^{* * * *}$ & $<0.001,<0.001$ & $<0.001(<0.001)^{* * * *}$ & $<0.001,<0.001$ \\
\hline $\begin{array}{l}\text { Covariance } \\
\text { (intercept, } \\
\text { month) }\end{array}$ & $0.002(<0.001) * * *$ & $0.002,0.003$ & $0.002(<0.001) * * *$ & $0.002,0.003$ & $0.002(<0.001) * * *$ & $0.001,0.003$ \\
\hline
\end{tabular}

Notes: $\mathrm{CI}=$ confidence interval. Higher scores on Turn 360 indicate more steps to turn (i.e., worse performance).

$* * * p<.001, * * p<.01, * p<.05$.

et al., 2010). The present study extends this work by demonstrating small training-related attenuation of declines in complex lower limb function across 10 years. More processing speed, memory, and reasoning training were associated with less decline in lower limb function over 10 years, suggesting that cognitive interventions training different cognitive domains can all positively impact physical function. This supports work that cognitive training mitigated declines in complex physical function (Ross, Sprague, et al., 2018; Smith-Ray et al., 2015, 2016). Although the mechanistic pathways by which this occurs are unknown, neuroimaging studies indicate compensation or neural dedifferentiation (Sleimen-Malkoun, Temprado, \& Hong, 2014), that is, decreased brain specialization in older adulthood due to impaired neural resource allocation, as potential explanations for the increased relatedness of physical and cognitive function in older adults. These processes are not interchangeable; compensation is an adaptive mechanism that should be associated with benefits on outcome performance (Cabeza et al., 2018), whereas dedifferentiation indicates neural impairment (Koen \& Rugg, 2019). While the current study is unable to identify which process is likelier to explain the current findings, there is evidence that prefrontal brain regions primarily responsible for cognitive processing speed (e.g., dorsolateral prefrontal cortex (Heuninckx, Wenderoth, Devaere, Peeters, \& Swinnen, 2005; Holtzer et al., 2014) are activated during walking tasks (Holtzer et al., 2014; Pelicioni et al., 2019). Since there are common brain regions underlying both cognitive and certain physical functions, training the implicated cognitive functions (e.g., processing speed) may impact the physical functions that recruit the same brain regions. Processing speed training does impact neurologic function and connectivity (Ross, Webb, et al., 2018), but it remains unknown whether these training-related changes are responsible for differences in complex physical function performance.

Despite evidence that grip strength and cognition are related in healthy older adults, cognitive training did not impact grip strength in this study. These results are similar to prior work that found grip strength decline was only attenuated in reasoning training after 5 years (Ross, Sprague, et al., 2018). Since weaker grip strength is associated with mortality (Granic et al., 2017), those who may be likeliest to receive benefits could have died during the follow-up period. The control participants with better grip strength who remained in the study may perform similarly to those with cognitive training. Relatedly, older adults healthy enough for in-person assessments after 10 years may be unlikely to be frail, so between-group differences may be more pronounced in frail older adults.

The second goal of this study was to evaluate baseline moderators of training effects. This study found that cognitive self-efficacy did not moderate training effects on any physical function outcome. This result is consistent with previous work in which processing speed training gains were not explained by cognitive self-efficacy (Sharpe et al., 2014). Although there were no differential dosage effects by cognitive self-efficacy, there was a significant interaction between baseline depressive symptoms and processing speed dosage across Turn 360 such that more depressive symptoms enhanced training-related attenuation of declines. That is, processing speed training benefitted complex physical function in those with more depressive symptoms. To the authors' knowledge, this has not been demonstrated elsewhere and suggests that there may 
be a relationship between depressive symptoms, processing speed, and complex physical function. Although the mechanistic pathway is unknown, processing speed training reduces depressive symptoms (Wolinsky, Mahncke, et al., 2009; Wolinsky, Vander Weg, et al., 2009), so it may be that the processing speed training "treated" depressive symptoms, in turn providing indirect benefits to physical function. It may also be the case that those with greater baseline depressive symptoms have a greater capacity for cognitive (and physical) improvement. There were generally larger standard deviations in baseline Turn 360 in those with greater depressive symptoms (data not shown), suggesting that those with more depressive symptoms may have greater capability to improve Turn 360 compared to those with fewer depressive symptoms since the maximum score was higher compared to those with lower depressive symptoms. Future research should replicate and extend these analyses to other functional outcomes. It may be that with additional training, those with greater depressive symptoms can perform as well as those with fewer depressive symptoms. Since there was a larger standard deviation in those with more depressive symptoms, this suggests those participants have an increased capacity for improvement.

The current results, however, do not suggest a mechanistic (i.e., mediation) pathway by which depressive symptoms, physical function, and cognitive training are related. Additionally, the design of the ACTIVE trial is not ideal to identify mechanistic pathways given the simultaneous assessment of possible mediators (e.g., changes in depressive symptoms) and outcomes (e.g., changes in physical function). This makes it difficult to determine the temporal ordering of the mechanism and outcome. Future work should design intervention studies that are appropriate for examining mechanistic pathways to elucidate why those with depressive symptoms conferred greater benefits. The current study was also unable to examine other proposed psychological mechanisms of transfer, such as engagement in cognitively engaging activities or physical activity. Future intervention studies should consider how engagement in cognitive training programs changes other everyday behaviors. It may be that something unique to the cognitive training program itself or whether mere engagement in an intervention program engenders behavior change elsewhere in the individual's daily life and is responsible for any training-related gains. However, this latter hypothesis has not been empirically tested.

Taken together, this study demonstrates the long-term association between more cognitive training sessions to complex lower limb function across 10 years. This study does not imply that cognitive training is the most effective intervention for attenuating age-related declines in these abilities as demonstrated by the small effect sizes. However, it is important to note that such effects, even if small, are still present after 10 years which is unique compared to other interventions. This work lends support that cognitive training programs are a plausible intervention modality for those who cannot or choose not to participate in physical-based interventions by capitalizing on the bidirectional cognitive-physical function relationship. It is noteworthy that the training gains were present 10 years later since the dosage compared to physical activity interventions was substantially smaller (e.g., 10 vs 49 hr; Oja et al., 2018), and the follow-up period was longer (e.g., 10 years vs immediate posttest or 2 years posttest max; Finnegan, Seers, \& Bruce, in press; Oja et al., 2018). The benefits of cognitive training were similar regardless of baseline cognitive self-efficacy and depressive symptoms (except processing speed training), providing further evidence that psychosocial beliefs are unlikely to impact cognitive training gains.

Future research should continue exploring the complex, dynamic relationship between physical and cognitive function with an emphasis on interventions that can simultaneously improve both health domains such as combined exercise-cognitive training programs (Marusic et al., 2018). Lastly, this work suggests that long-term benefits may be greater for at-risk compared to low-risk samples. Cognitive, especially speed of processing, training may be more effective as a targeted intervention rather than a universal intervention for older adults regardless of physical functional ability. The ACTIVE sample performed substantially better on the Turn 360 task compared to older adults at risk for falls (i.e., around 6 steps vs $>12$ steps; Lipsitz, Jonsson, Kelley, \& Koestner, 1991). Accumulating evidence suggests that cognitive training, particularly speed of processing, may be especially effective for high-risk samples across a breadth of outcomes (e.g., Ball, Edwards, \& Ross, 2007; Ball, Edwards, Ross, \& McGwin, 2010; Ball, Ross, Roth, \& Edwards, 2013; Edwards, Fausto, Tetlow, Corona, \& Valdés, 2018; Ross et al., 2016, 2017; Smith-Ray et al., 2015; Sprague et al., 2019). Identifying effective, time-efficient interventions-especially for high-risk samples-are important to promote older adults' health, independence, and well-being.

\section{Supplementary Material}

Supplementary data are available at The Journals of Gerontology, Series B: Psychological Sciences and Social Sciences online.

Supplementary Table 1. Raw means (standard deviations) of grip strength and Turn 360 across all timepoints by intervention group.

\section{Funding}

This work was supported by cooperative agreements from the National Institute on Aging (U01AG14260, U01AG14263, U01AG14276, U01AG14282, U01AG14289); and the National Institute of Nursing Research (U01NR04507, U01NR04508). B. N. Sprague also received additional support from the Albert and Lorraine Kligman Graduate Fellowship through The College of Health and Human Development, The Pennsylvania State University. 


\section{Acknowledgments}

Special thanks to Drs. Alyssa Gamaldo, Stephanie Lanza, and David Proctor for their feedback on prior drafts of this document. Data used for these analyses are publicly available through the Inter-University Consortium for Political and Social Research (https://www.icpsr.umich.edu/ icpsrweb/NACDA/studies/36036). Syntax or additional study materials are available upon request by contacting the corresponding author (B. N. Sprague). All authors have complied with the APA ethical standards of research in regards to this submission.

\section{Conflict of Interest}

None reported.

\section{References}

Azadian, E., Majlesi, M., \& Jafarnezhadgero, A. A. (2018). The effect of working memory intervention on the gait patterns of the elderly. Journal of Bodywork and Movement Therapies, 22(4), 881-887. doi:10.1016/j.jbmt.2017.08.008

Azadian, E., Torbati, H. R., Kakhki, A. R., \& Farahpour, N. (2016). The effect of dual task and executive training on pattern of gait in older adults with balance impairment: A randomized controlled trial. Archives of Gerontology and Geriatrics, 62, 83-89. doi:10.1016/j.archger.2015.10.001

Ball, K., Edwards, J. D., \& Ross, L. A. (2007). The impact of speed of processing training on cognitive and everyday functions. The Journals of Gerontology, Series B: Psychological Sciences and Social Sciences, 62 (Spec No. 1), 19-31. doi:10.1093/geronb/62. special_issue_1.19

Ball, K., Edwards, J. D., Ross, L. A., \& McGwin, G. Jr. (2010). Cognitive training decreases motor vehicle collision involvement of older drivers. Journal of the American Geriatrics Society, 58(11), 2107-2113. doi:10.1111/j.1532-5415.2010.03138.x

Ball, K. K., Ross, L. A., Roth, D. L., \& Edwards, J. D. (2013). Speed of processing training in the ACTIVE study: how much is needed and who benefits? Journal of Aging and Health, 25(Suppl. 8), 65S-84S. doi:10.1177/0898264312470167

Beaudoin, M., \& Desrichard, O. (2017). Memory self-efficacy and memory performance in older adults: The mediating role of task persistence. Swiss Journal of Psychology, 76(1), 23-33. doi:10.1024/1421-0185/a000188

Briggs, R., Carey, D., Kenney, R. A., \& Kennelly, S. P. (2018). What is the longitudinal relationship between gait abnormalities and depression in a cohort of community-dwelling older people? Data from the Irish Longitudinal Study on Ageing (TILDA). The American Journal of Geriatric Psychiatry, 26(1), 75-86. doi:10.1016/j.jagp.2017.08.012

Buchman, A. S., Wilson, R. S., Yu, L., Boyle, P. A., Bennett, D. A., \& Barnes, L. L. (2016). Motor function is associated with incidence disability in older African Americans. The Journals of Gerontology, Series A: Biological Sciences and Medical Sciences, 71(5), 696-702. doi:10.1093/gerona/glv186

Cabeza, R., Albert, M., Belleville, S., Craik, F. I. M., Duarte, A., Grady, C. L., ... Rajah, M. N. (2018). Maintenance, reserve and compensation: The cognitive neuroscience of healthy ageing. Nature Reviews. Neuroscience, 19(11), 701-710. doi:10.1038/ s41583-018-0068-2

Cesari, M., Penninx, B. W., Pahor, M., Lauretani, F., Corsi, A. M., Rhys Williams, G., ... Ferrucci, L. (2004). Inflammatory markers and physical performance in older persons: The InCHIANTI study. The Journals of Gerontology, Series A: Biological Sciences and Medical Sciences, 59(3), 242-248. doi:10.1093/gerona/59.3.m242

Choi, K., Jeon, G.-S., \& Cho, S.-I. (2017). Prospective study on the impact of fear of falling on functional decline among community dwelling elderly women. International Journal of Environmental Research and Public Health, 14(5), 469. doi:10.3390/ ijerph14050469

Chou, C. H., Hwang, C. L., \& Wu, Y. T. (2012). Effect of exercise on physical function, daily living activities, and quality of life in the frail older adults: A meta-analysis. Archives of Physical Medicine and Rehabilitation, 93(2), 237-244. doi:10.1016/j.apmr.2011.08.042

Edwards, J. D., Fausto, B. A., Tetlow, A. M., Corona, R. T., \& Valdés, E. G. (2018). Systematic review and meta-analyses of useful field of view cognitive training. Neuroscience and Biobehavioral Reviews, 84, 72-91. doi:10.1016/j. neubiorev.2017.11.004

Edwards, J. D., Xu, H., Clark, D. O., Guey, L. T., Ross, L. A., \& Unverzagt, F. (2017). Speed of processing training results in lower risk of dementia. Alzheimer's \& Dementia: Translational Research \& Clinical Interventions, 3(4), 603-611. doi:10.1016/j. trci.2017.09.002

Finnegan, S., Seers, K., \& Bruce, J. (In press). Long-term follow-up of exercise interventions aimed at preventing falls in older people living in the community: A systematic review and meta-analysis. Physiotherapy. 105(2), 187-199. doi:10.1016/j. physio.2018.09.002

Gill, T. M., Williams, C. S., \& Tinetti, M. E. (1995). Assessing risk for the onset of functional dependence among older adults: The role of physical performance. Journal of the American Geriatrics Society, 43(6), 603-609. doi:10.1111/j.1532-5415.1995. tb07192.x

Gobbens, R. J., \& van Assen, M. A. (2014). The prediction of ADL and IADL disability using six physical indicators of frailty: A longitudinal study in the Netherlands. Current Gerontology and Geriatrics Research, 2014, 358137. doi:10.1155/2014/358137

Granic, A., Davies, K., Jagger, C., Dodds, R. M., Kirkwood, T. B. L., \& Sayer, A. A. (2017). Initial level and rate of change in grip strength predict all-cause mortality in very old adults. Age and Ageing, 46(6), 970-976. doi:10.1093/ageing/afx087

Henderson, R. M., Miller, M. E., Fielding, R. A., Gill, T. M., Glynn, N. W., Guralnik, J. M., . . Kritchevsky, S. B. (2018). Maintenance of physical function 1 year after exercise intervention in at-risk older adults: Follow-up from the LIFE Study. The Journals of Gerontology, Series A: Biological Sciences and Medical Sciences, 73(5), 688-694. doi:10.1093/ gerona/glx231

Heuninckx, S., Wenderoth, N., Devaere, F., Peeters, R., \& Swinnen, S. P. (2005). Neural basis of aging: The penetration of cognition into action control. The Journal of Neuroscience, 25(29), 6787-6796. doi:10.1523/JNEUROSCI.1263-05.2005

Holtzer, R., Epstein, N., Mahoney, J. R., Izzetoglu, M., \& Blumen, H. M. (2014). Neuroimaging of mobility in aging: 
A targeted review. The Journals of Gerontology, Series A: Biological Sciences and Medical Sciences, 69(11), 1375-1388. doi:10.1093/gerona/glu052

Jobe, J. B., Smith, D. M., Ball, K., Tennstedt, S. L., Marsiske, M., Willis, S. L., ... Kleinman, K. (2001). ACTIVE: A cognitive intervention trial to promote independence in older adults. Controlled Clinical Trials, 22(4), 453-479. doi:10.1016/ s0197-2456(01)00139-8

Keysor, J. J., \& Brembs, A. (2011). Exercise: Necessary but not sufficient for improving function and preventing disability? Current Opinion in Rheumatology, 23(2), 211-218. doi:10.1097/ BOR.0b013e3283432c41

Koen, J. D., \& Rugg, M. D. (2019). Neural dedifferentiation in the aging brain. Trends in Cognitive Sciences, 23(7), 547-559. doi:10.1016/j.tics.2019.04.012

Kronzer, V. L., Jerry, M. R., Ben Abdallah, A., Wildes, T. S., Stark, S. L., McKinnon, S. L., ... Avidan, M. S. (2016). Preoperative falls predict postoperative falls, functional decline, and surgical complications. EBioMedicine, 12, 302-308. doi:10.1016/j.ebiom.2016.08.039

Lachman, M. E., Baltes, P., Nesselroade, J. R., \& Willis, S. L. (1982). Examination of personality-ability relationships in the elderly: The role of the contextual (interface) assessment mode. Journal of Research in Personality, 16(4), 485-501. doi:10.1016/0092-6566(82)90007-1

Li, K. Z., Roudaia, E., Lussier, M., Bherer, L., Leroux, A., \& McKinley, P. A. (2010). Benefits of cognitive dual-task training on balance performance in healthy older adults. The Journals of Gerontology, Series A: Biological Sciences and Medical Sciences, 65(12), 1344-1352. doi:10.1093/gerona/glq151

Lipsitz, L. A., Jonsson, P. V., Kelley, M. M., \& Koestner, J. S. (1991). Causes and correlates of recurrent falls in ambulatory frail elderly. The Journals of Gerontology, Series A: Biological Sciences and Medical Sciences, 46(4), M114-M122. doi:10.1093/geronj/46.4.M114

Lohman, M. C., Rebok, G. W., Spira, A. P., Parisi, J. M., Gross, A. L., \& Kueider, A. M. (2013). Depressive symptoms and memory performance among older adults: Results from the ACTIVE memory training intervention. Journal of Aging and Health, 25(Suppl. 8), 209S-229S. doi:10.1177/0898264312460573

Mangani, I., Cesari, M., Russo, A., Onder, G., Maraldi, C., Zamboni, V., ... Landi, F. (2008). Physical function, physical activity and recent falls. Results from the "Invecchiamento e Longevità nel Sirente (ilSIRENTE)" Study. Aging Clinical and Experimental Research, 20(3), 234-241. doi:10.1007/BF03324778

Marusic, U., \& Grosprêtre, S. (2018). Non-physical approaches to counteract age-related functional deterioration: Applications for rehabilitation and neural mechanisms. European Journal of Sport Science, 18(5), 639-649. doi:10.1080/17461391.2018.1447018

Marusic, U., Kavcic, V., Giordani, B., Gerževič, M., Meeusen, R., \& Pišot, R. (2015). Computerized spatial navigation training during 14 days of bed rest in healthy older adult men: Effect on gait performance. Psychology and Aging, 30(2), 334-340. doi:10.1037/pag0000021

Marusic, U., Verghese, J., \& Mahoney, J. R. (2018). Cognitive-based interventions to improve mobility: A systematic review and metaanalysis. Journal of the American Medical Directors Association, 19(6), 484-491.e3. doi:10.1016/j.jamda.2018.02.002

Mujika, I., \& Padilla, S. (2000). Detraining: Loss of training-induced physiological and performance adaptations. Part I: Short-term insufficient training stimulus. Sports Medicine, 30(2), 79-87. doi:10.2165/00007256-200030020-00002

Murphy, S. L., Dubin, J. A., \& Gill, T. M. (2003). The development of fear of falling among community-dwelling older women: Predisposing factors and subsequent fall events. The Journals of Gerontology, Series A: Biological Sciences and Medical Sciences, 58(10), M943-M947. doi:10.1093/gerona/58.10.M943

Ng, T. P., Feng, L., Nyunt, M. S., Feng, L., Niti, M., Tan, B. Y., ... Yap, K. B. (2015). Nutritional, physical, cognitive, and combination interventions and frailty reversal among older adults: A randomized controlled trial. The American Journal of Medicine, 128(11), 1225-1236.e1. doi:10.1016/j. amjmed.2015.06.017

Nofuji, Y., Shinkai, S., Taniguchi, Y., Amano, H., Nishi, M., Murayama, H., ... Suzuki, T. (2016). Associations of walking speed, grip strength, and standing balance with total and causespecific mortality in a general population of Japanese elders. Journal of the American Medical Directors Association, 17(2), 184.e1-184.e7. doi:10.1016/j.jamda.2015.11.003

Oja, P., Kelly, P., Murtagh, E. M., Murphy, M. H., Foster, C., \& Titze, S. (2018). Effects of frequency, intensity, duration and volume of walking interventions of CVD risk factors: A systematic review and meta-regression analysis of randomized controlled trials among inactive healthy adults. British Journal of Sports Medicine, 52(12), 769-775. doi:10.1136/bjsports-2017-098558

Paterson, D. H., \& Warburton, D. E. (2010). Physical activity and functional limitations in older adults: A systematic review related to Canada's Physical Activity Guidelines. The International Journal of Behavioral Nutrition and Physical Activity, 7, 38. doi:10.1186/1479-5868-7-38

Payne, B. R., Jackson, J. J., Hill, P. L., Gao, X., Roberts, B. W., \& Stine-Morrow, E. A. (2012). Memory self-efficacy predicts responsiveness to inductive reasoning training in older adults. The Journals of Gerontology, Series B: Psychological Sciences and Social Sciences, 67(1), 27-35. doi:10.1093/geronb/gbr073

Pelicioni, P. H. S., Tijsma, M., Lord, S. R., \& Menant, J. (2019). Prefrontal cortical activation measured by fNIRS during walking: Effects of age, disease and secondary task. PeerJ, 7, e6833. doi:10.7717/peerj.6833

Radloff, L. S. (1977). The CES-D scale: A self-report depression scale for research in the general population. Applied Psychological Measurement, 1, 385-401. doi:10.1177/014662167700100306

Rebok, G. W., Ball, K. K., Guey, L. T., Jones, R. N., Kim, H.-Y., King, J. W., . . . Willis, S. L. (2014). Ten year effects of the Advanced Cognitive Training for Independent and Vital Elderly cognitive training trial on cognition and everyday functioning in older adults. Journal of the American Geriatrics Society, 62, 16-24. doi:10.1111/jgs.12607

Ross, L. A., Edwards, J. D., O’Connor, M. L., Ball, K. K., Wadley, V. G., \& Vance, D. E. (2016). The transfer of cognitive speed of processing training to older adults' driving mobility across 5 years. The Journals of Gerontology, Series B: Psychological Sciences and Social Sciences, 71(1), 87-97. doi:10.1093/geronb/gbv022

Ross, L. A., Freed, S. A., Edwards, J. D., Phillips, C. B., \& Ball, K. (2017). The impact of three cognitive training programs on driving cessation across 10 years: A randomized controlled trial. The Gerontologist, 57(5), 838-846. doi:10.1093/geront/gnw143 
Ross, L. A., Sprague, B. N., Phillips, C. B., O’Connor, M. L., \& Dodson, J. E. (2018). The impact of three cognitive training interventions on older adults' physical functioning across 5 years. Journal of Aging and Health, 30(3), 475-498. doi:10.1177/0898264316682916

Ross, L. A., Webb, C. E., Whitaker, C., Hicks, J. M., Schmidt, E. L., Samimy, S., . . . Visscher, K. M. (2018). The effects of useful field of view training on brain activity and connectivity. Journals of Gerontology, Series B: Psychological Sciences and Social Sciences. 74(7), 1152-1162. doi:10.1093/geronb/gby041

Sharpe, C., Holup, A. A., Hansen, K. E., \& Edwards, J. D. (2014). Does self-efficacy affect responsiveness to cognitive speed of processing training? Journal of Aging and Health, 26(5), 786806. doi:10.1177/0898264314531615

Sleimen-Malkoun, R., Temprado, J. J., \& Hong, S. L. (2014). Aging induced loss of complexity and dedifferentiation: Consequences for coordination dynamics within and between brain, muscular and behavioral levels. Frontiers in Aging Neuroscience, 6, 140. doi:10.3389/fnagi.2014.00140

Smith-Ray, R. L., Hughes, S. L., Prohaska, T. R., Little, D. M., Jurivich, D. A., \& Hedeker, D. (2015). Impact of cognitive training on balance and gait in older adults. The Journals of Gerontology, Series B: Psychological Sciences and Social Sciences, 70(3), 357-366. doi:10.1093/geronb/gbt097

Smith-Ray, R. L., Irmiter, C., \& Boulter, K. (2016). Cognitive training among cognitively impaired older adults: A feasibility study assessing the potential improvement in balance. Frontiers in Public Health, 4, 219. doi:10.3389/fpubh.2016.00219

Smith-Ray, R. L., Makowski-Woidan, B., \& Hughes, S. L. (2014). A randomized trial to measure the impact of a community-based cognitive training intervention on balance and gait in cognitively intact black older adults. Health Education \& Behavior, 41(Suppl. 1), 62S-69S. doi:10.1177/1090198114537068

Spedden, M. E., Malling, A. S. B., Andersen, K. K., \& Jensen, B. R. (2017). Association between gross-motor and executive function depends on age and motor task complexity. Developmental Neuropsychology, 42(7-8), 495-506. doi:10.1080/87565641.2017.1399129

Sprague, B. N., Freed, S. A., Webb, C. E., Phillips, C. B., Hyun, J., \& Ross, L. A. (2019). The impact of behavioral interventions on cognitive function in healthy older adults: A systematic review. Ageing Research Reviews, 52, 32-52. doi:10.1016/j.arr.2019.04.002

Steinhagen-Thiessen, E., \& Borchelt, M. (1999). Morbidity, medication, and functional limitations in very old age. In P. B. Baltes \& K. U. Mayer (Eds.), The Berlin Aging Study: Aging from 70 to 100 (pp. 131-166). Cambridge, UK: Cambridge University Press.

Tager, I. B., Swanson, A., \& Satariano, W. A. (1998). Reliability of physical performance and self-reported functional measures in an older population. The Journals of Gerontology, Series A: Biological Sciences and Medical Sciences, 53(4), M295-M300. doi:10.1093/gerona/53a.4.m295

van Aalst, M. J., Oosterhof, J., Nijhuis-van der Sanden, M. W., \& Schreurs, B. W. (2014). Can the length of hospital stay after total hip arthroplasty be predicted by preoperative physical function characteristics? American Journal of Physical Medicine \& Rehabilitation, 93(6), 486-492. doi:10.1097/ PHM.0000000000000054

Verghese, J., Mahoney, J., Ambrose, A. F., Wang, C., \& Holtzer, R. (2010). Effect of cognitive remediation on gait in sedentary seniors. The Journals of Gerontology, Series A: Biological Sciences and Medical Sciences, 65(12), 1338-1343. doi:10.1093/gerona/ glq127

Wolinsky, F. D., Mahncke, H. W., Weg, M. W., Martin, R., Unverzagt, F. W., Ball, K. K., ... Tennstedt, S. L. (2009). The ACTIVE cognitive training interventions and the onset of and recovery from suspected clinical depression. The Journals of Gerontology, Series B: Psychological Sciences and Social Sciences, 64(5), 577-585. doi:10.1093/geronb/gbp061

Wolinsky, F. D., Vander Weg, M. W., Martin, R., Unverzagt, F. W., Ball, K. K., Jones, R. N., \& Tennstedt, S. L. (2009). The effect of speed-of-processing training on depressive symptoms in ACTIVE. The Journals of Gerontology, Series A: Biological Sciences and Medical Sciences, 64(4), 468-472. doi:10.1093/ gerona/gln044

Zahodne, L. B., Watson, C. W., Seehra, S., \& Martinez, M. N. (2018). Positive psychosocial factors and cognition in ethnically diverse older adults. Journal of the International Neuropsychological Society, 24(3), 294-304. doi:10.1017/S1355617717000935 